\title{
Role of Transcranial Ultrasound Augmented by Color Doppler Study in Evaluation Neonatal Brain Problem in Preterm Patient
}

\author{
Ahmed F. Yousef, Hamada M. Khater, Shiamaa H. Abdel Bedea
}

Department of Radiology, Benha faculty of medicine, Benha University, Egypt.

Correspondence to: Shiamaa H. Abdel Bedea, Department of Radiology, Benha faculty of medicine, Benha University, Egypt.

Email:

shiamaa.hamouda@gmail.com

Received: 15 January 2021

Accepted: 17 February 2021

\begin{abstract}
:
Background: It is well known that the number of surviving preterm infants is today steadily rising. In spite of the improvements in perinatal medicine, brain injury is still a major clinical problem and remains an important cause of perinatal morbidity and mortality. The main aim to assess the significance of universal cranial ultrasound screening in all preterm neonates in
\end{abstract} the neonatal ICU with gestational age between 28 and 37 weeks of gestational age even if clinically silent. Methods: One hundred (100) preterm neonates were examined after delivery by 3-7 days by cranial Doppler ultrasound at radiology department of Benha Children Hospital (BENCH). Fifty two of them were male patients and 48 were females. The age ranged from 28 to 37 weeks (early neonate from 28 to 32 weeks and moderate \& late neonate from 32 to 37 weeks, using two transducer probes, convex Array transducer $(6 \mathrm{MHz})$ and Linear Array transducer $(7.5 \mathrm{MHz})$. The anterior fontanel was used as the principal acoustic window. The trans-mastoid view was additionally used particularly to assess the posterior fossa structures. Results: Through the scheduled study period, one hundred (100) preterm neonates were examined by convex array transducer and linear array transducer, the anterior fontanel was used as the principal acoustic window, 52\% of them were male patients and $48 \%$ were female patients, with age ranging from 28 to 37 weeks classified as group I $<32$ weeks represent $44 \%$ and group II $\geq 32$ weeks represent 56\%. Conclusion: Cranial ultrasound was proven to have a significant role in evaluation neonatal brain problems in preterm patients while most of abnormal brain scans are usually asymptomatic patients.

Keywords: Cranial ultrasound; neonatal brain problems; preterm neonates 


\section{Introduction}

Cranial ultrasound is a safe imaging modality that does not need sedation and can be performed bedside. It can be reiterated as often as requisite because of the lack of ionizing radiation (1). It is inexpensive, repeatable, and allows continuous bedside monitoring of CBFV, which is especially useful in the intensive care setting (2).

Transcranial Doppler ultrasonography (TCD) is the only noninvasive examination method that enables the reliable evaluation of blood flow from the basal intracerebral vessels, adding physiologic information to the anatomic images. TCD is comparatively inexpensive, can be done at bedside, and allows monitoring in acute emergency settings and for prolonged periods with a high temporal resolution, making it idealistic for studying dynamic cerebrovascular responses (3).

The importance of preterm screening by cranial ultrasound is sustained by the observation that in this vulnerable group, babies who are found to have abnormal brain scans are usually asymptomatic. Only occasionally these patients develop symptoms (seizures or other neurological symptoms) because of a massive intracranial hemorrhage (ICH) (4). The preterm neonates. It can reliably detect germinal matrix, intraventricular hemorrhage, cystic periventricular leukomalacia, ventricular dilatation, and post-hemorrhagic hydrocephalus (5). Congenital brain anomalies could be seen during the cranial ultrasound screening, such as Dandy-Walker malformation (DWM), Chiari II malformation, agenesis of corpus callosum (1).

TCD examinations have acquired a significant role in the very early phase of critical cerebral pathologies, also during follow-up of patients with chronic CVDs. As well as it is useful on both adults and children to diagnose and monitor vasospasm (VSP) after subarachnoid hemorrhage $(\mathrm{SAH})$ of various etiologies (aneurysm rupture and traumatic brain injury $[\mathrm{TBI}]$ ), and cerebral hemodynamic changes after stroke including cryptogenic stroke (6). The final outcome is not only the major distress incurred by the newborn and later the child and adult, but also the great burden inflicted on both their families and the national health system (7). 
The main objective of study was to describe indications and techniques for cranial US for any preterm neonates to assess neonatal brain problems, and to describe and explain their imaging appearance.

\section{Patients and method}

This is a retrospective study aiming to emphasize the role of cranial ultrasound examination in preterm neonates, the study was conducted on all preterm (100 patient) in the radiology department of Benha Children Hospital (BENCH), between 28 and 37 weeks gestational age, between the third and seventh day of life, from February 2019 to December 2020.

The population of the study was classified in to two groups according to their age:

○ Group (I): Babies born before 32 weeks are called early preterm according to the definition of the American college of obstetricians and gynecologists.

- Group (II): Babies born between 32 and 37 weeks of pregnancy are considered moderate and late preterm.

Screening cranial ultrasonography was obtained by the third and not later than the seventh day of life in all newborns. It is then reiterated based on the gestational age and the results of the test. Specially, patients with PVL, even with grade I, have at least one cranial ultrasonography followup after 7-10 days. Clinical data of the children were retrospectively obtained from the Neonatology and Neonatal Intensive Care Unit information system database.

In preparation for performing neonatal CUS proper antiseptic precautions were undertaken because of the poor immune system of neonates, particularly those born premature. Correct hand washing and disinfection of the transducer were done before performing the study to decrease transmission of infectious agents to the immunocompromised newborn.

Precautions were done to keep the neonate's body temperature, as the newborn is liable to rapid heat loss by keeping the baby covered, shorten the study time and rapid removal of ultrasound gel from the scan area of the neonate head instantly after finishing the exam to avoid heat loss.

Pressure over the anterior fontanelle was avoided, in particular in a critically ill premature neonate. 
Cranial ultrasonography was performed with two transducer probes, Convex Array transducer (6 MHz) and Linear Array transducer $(7.5 \mathrm{MHz})$. The anterior fontanel was used as the main acoustic window. The trans-mastoid view was additionally used especially to evaluate the posterior fossa structures.

The scanning included:

A. Five standard coronal planes:

- Frontal lobes and roof of the orbits.

- Frontal horns of the lateral ventricles.

- Foramen of Monroe and the third ventricle.

- Body of the lateral ventricles.

- Occipital lobes.

B. Five standard sagittal planes:

- Mid-sagittal plane.

- Left and right parasagittal planes through lateral ventricles (caudothalamic view).

- Left and right parasagittal planes through insula.

\section{Ethical committee approval}

- Approval of the ethics committee of researchers of Benha University.

- An informed consent was done from all the participants before taking any data or doing any imaging techniques.
- The consent contained:

1. Simple explanation of the aim of the study to the patients.

2. No harmful maneuvers has performed or used.

3. All data is considered confidential.

4. All samples has used in research only.

5. All participants has informed by the results of the study.

6. Signatures or fingerprints of the participants has taken.

\section{Statistical analysis:}

Data were statistically described in terms of frequencies and percentages. Comparison was done between the different modalities using McNemar test. Agreement was done using kappa statistic. Accuracy was represented using the terms, +ve predictive value, -ve predictive value, and overall accuracy. $\mathrm{P}$ values less than 0.05 was considered statistically significant. All statistical calculations were done using computer program IBM SPSS (Statistical Package for the Social Science; IBM Corp, Armonk, NY, USA) release 22 for Microsoft Windows.

\section{Results}

Throughout the study period (one year), one hundred (100) preterm neonates were 
examined after delivery with 3-7 days by cranial Doppler ultrasound at radiology department of Benha Children Hospital $(\mathrm{BENCH})$. Fifty two of them were male patients and 48 were females. The age ranging from 28 to 37 weeks (early neonate from 28 to 32 weeks and moderate $\&$ late neonate from 32 to 37 weeks).

In our study, we proved that preterm neonates less than 37 weeks of gestational age are more susceptible to hemorrhagic and ischemic brain lesions because of the immaturity of capillary bed, cerebral autoregulation and vascular anastomosis, with consequent adverse outcome, poor cognitive development, and cerebral palsy.

Table 1: Distribution of the studied groups according to sex male patient represent $52 \%$ and female patient represent $48 \%$.

Table 2: Distribution of the studied groups according to prematurity, group I (>32 weeks) represent $44 \%$ and group II ( $\geq 32$ weeks) represent $56 \%$.

Table 3: Distribution of the studied groups according to history of risky pregnancy and birth insult.

Table 4: Distribution of the studied groups according to neurological symptoms.
Figure 1: Distribution of the studied groups according to Doppler results, $20 \%$ represent abnormal results and $80 \%$ represent normal doppler US examination.

Table 5: There is significance difference between age groups regarding to results of TCUS, as $\mathrm{P}$ value $>0.05$.

Table 6: Comparison between the studied groups according to IVH and PVL, there is significance statistical value in diagnosis of PVL as $\mathrm{P}$ value $>0.05$ and none significance statistical value in $\mathrm{IVH}$ as $\mathrm{P}$ value $<0.05$

Figure 2: Distribution of the studied groups according to IVH and PVL.

\section{Cases}

Case 1 - Figure 3: Shows dilated ventricular system full off echogenic hemorrhage seen obliterating both frontal horns and occipital horns of lateral ventricles, with hypoechoic patches diffuse at brain parenchyma with high resistive index on applying color Doppler. These findings demonstrating IVH grade III \& cerebral hemorrhage.

Case 2 - Figure 4: Shows multiple variable sized septated cystic lesions with 
turbid fluid content surrounding both ventricles with low resistive index on applying color doppler. These findings demonstrating PVL grade III.

Case 3 - Figure 5: Shows bilateral thin wall simple cyst with clear fluid seen at both caudothalamic grooves with normal color Doppler evaluation (RI 0.7) most likely anterior choroidal simple Cyst.
Case 4 - Figure 6: Shows normal parenchymal echo-pattern of both cerebral hemisphere, normal appearance of ventricular system, no evidence of intracranial, intra ventricular He \& no focal lesion is noted whether solid or cystic, no deformity or midline shift, no evidence of PVL, no evidence of extra axial fluid collection, normal appearance of posterior cranial fossa, normal color doppler evaluation.

Table 1: Distribution of the studied groups according to sex

\begin{tabular}{ccc}
\hline Sex & Value & \\
& No (100) & \% \\
\hline Male & 52 & 52 \\
Female & 48 & 48 \\
\hline
\end{tabular}

Table 2: Distribution of the studied groups according to prematurity

\begin{tabular}{ccc}
\hline Types & Value \\
& No (100) & \% \\
\hline Group I $(<32 \mathrm{w})$ & 44 & 44 \\
Group II $(\geq 32 \mathrm{w})$ & 56 & 56 \\
\hline
\end{tabular}

Table 3: Distribution of the studied groups according to history of risky pregnancy and birth insult

\begin{tabular}{ccc}
\hline & Value & \\
& No (100) & \% \\
\hline Risky pregnancy & & \\
Risky & 46 & 46 \\
None & 54 & 54 \\
Birth insult & & \\
Yes & 4 & 4 \\
No & 96 & 96 \\
\hline
\end{tabular}


Table 4: Distribution of the studied groups according to neurological symptoms

\begin{tabular}{lcc}
\hline & Value & \\
& No (100) & \% \\
\hline Neural symptoms & 14 & 14 \\
Present & 86 & 86 \\
Absent & 6 & 6 \\
Convulsions, poor suckling & 2 & 2 \\
Craniostenosis & 6 & 6 \\
Week suckling, poor crying & 86 & 86 \\
None & & \\
\hline
\end{tabular}

Table 5: Comparison between the studied groups according to results of TCUS

\begin{tabular}{ccccccc}
\hline & \multicolumn{2}{c}{ Group I (44) } & \multicolumn{2}{c}{ Group II (56) } & Statistical & \multirow{2}{*}{ P value } \\
Results of TCUS & No & $\mathbf{\%}$ & No & \% & & \\
& 22 & 50.0 & 10 & 17.9 & \multirow{2}{*}{11.70} & \multirow{2}{*}{$0.001^{* *}$} \\
\hline Abnormal & 22 & 50.0 & 46 & 82.1 & & \\
Normal & & & & &
\end{tabular}

Table 6: Comparison between the studied groups according to IVH and PVL

\begin{tabular}{|c|c|c|c|c|c|c|}
\hline & \multicolumn{2}{|c|}{ Group I (44) } & \multicolumn{2}{|c|}{ Group II (56) } & \multirow{2}{*}{ Statistical test (x2) } & \multirow{2}{*}{$P$ value } \\
\hline & No & $\%$ & No & $\%$ & & \\
\hline \multicolumn{7}{|l|}{ IVH } \\
\hline Yes & 6 & 13.6 & 6 & 10.7 & \multirow{3}{*}{0.20} & \multirow{3}{*}{0.66} \\
\hline No & 38 & 86.4 & 50 & 89.3 & & \\
\hline \multicolumn{5}{|l|}{ PVL } & & \\
\hline Yes & 8 & 18.2 & 2 & 3.6 & \multirow{2}{*}{$\mathrm{FET}=4.33$} & \multirow{2}{*}{$0.02 *$} \\
\hline No & 36 & 81.8 & 54 & 96.4 & & \\
\hline
\end{tabular}

\section{Doppler results}

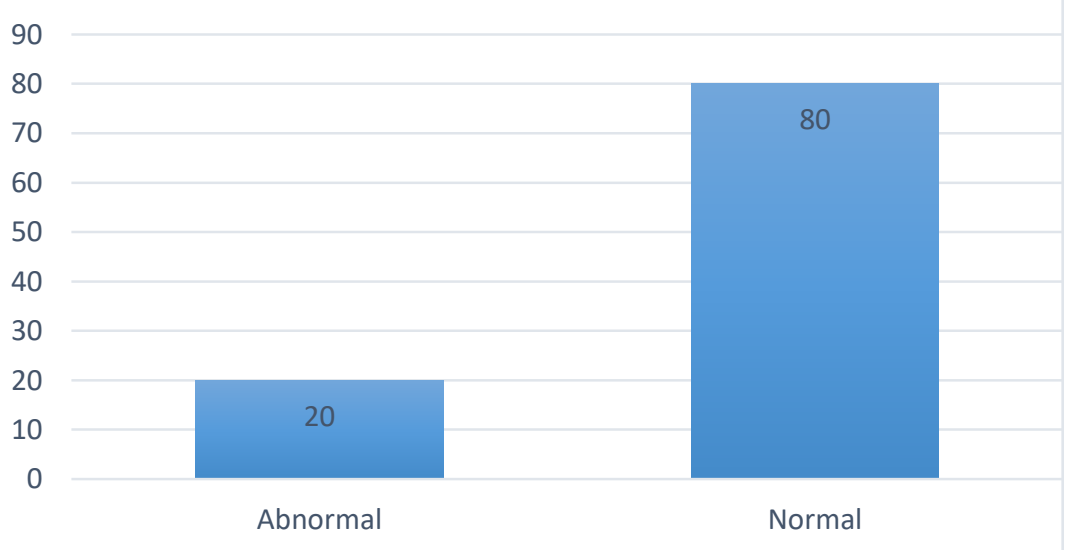

Fig (1): Distribution of the studied groups according to Doppler results 


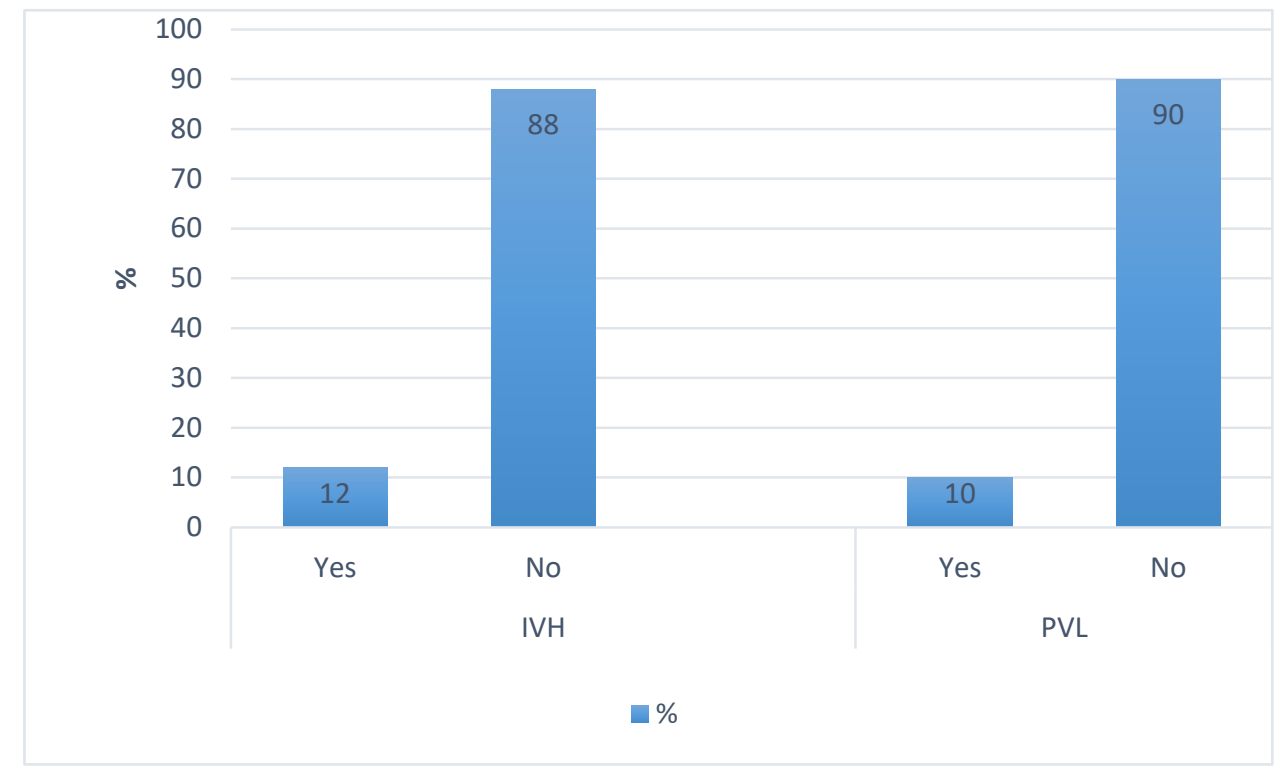

Fig (2): Distribution of the studied groups according to IVH and PVL
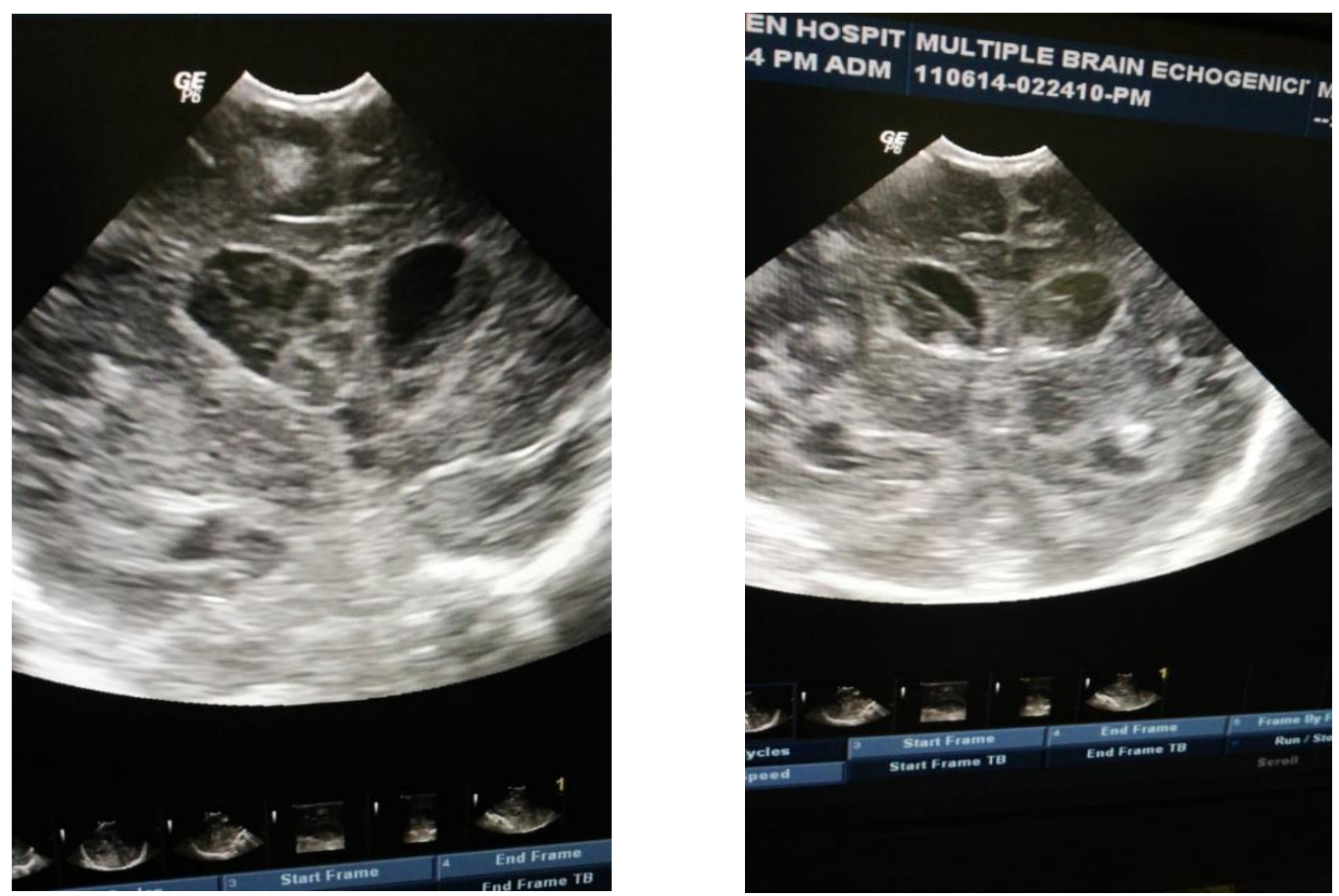

Fig (3) - Case 1: Bilateral IVH grade III and cerebral Hge 

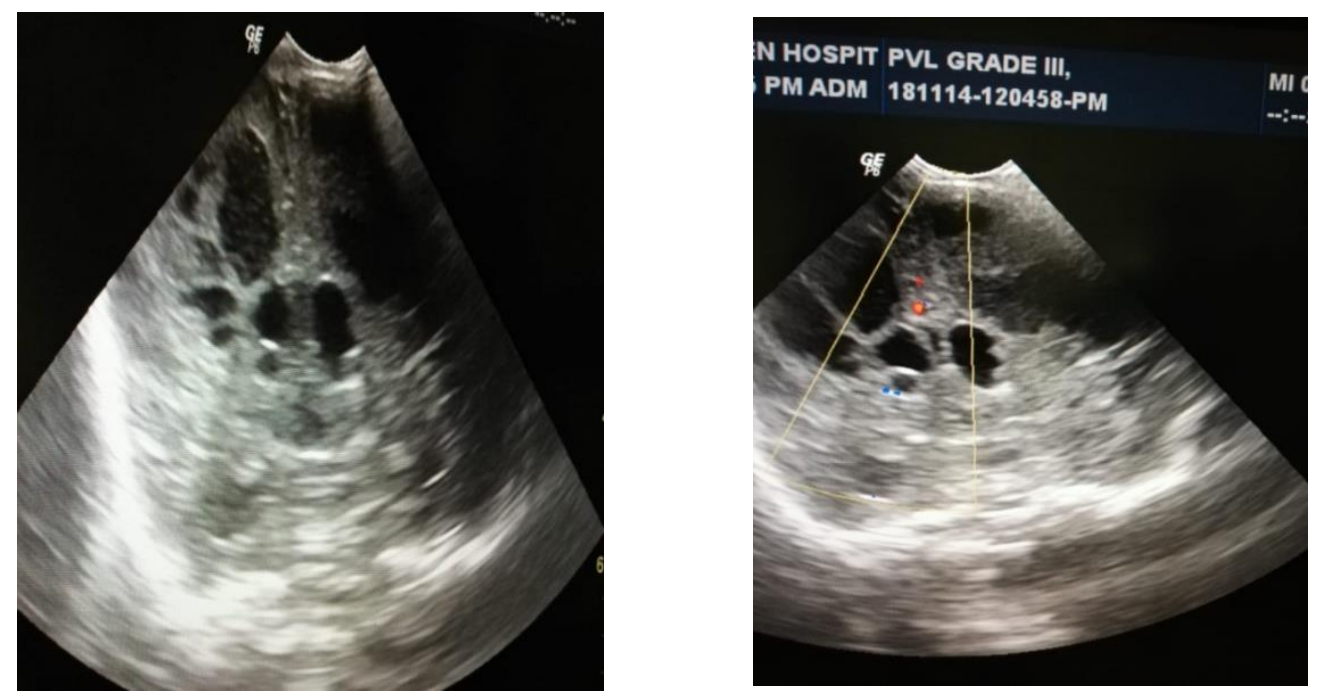

Fig (4) - Case 2: PVL grade III
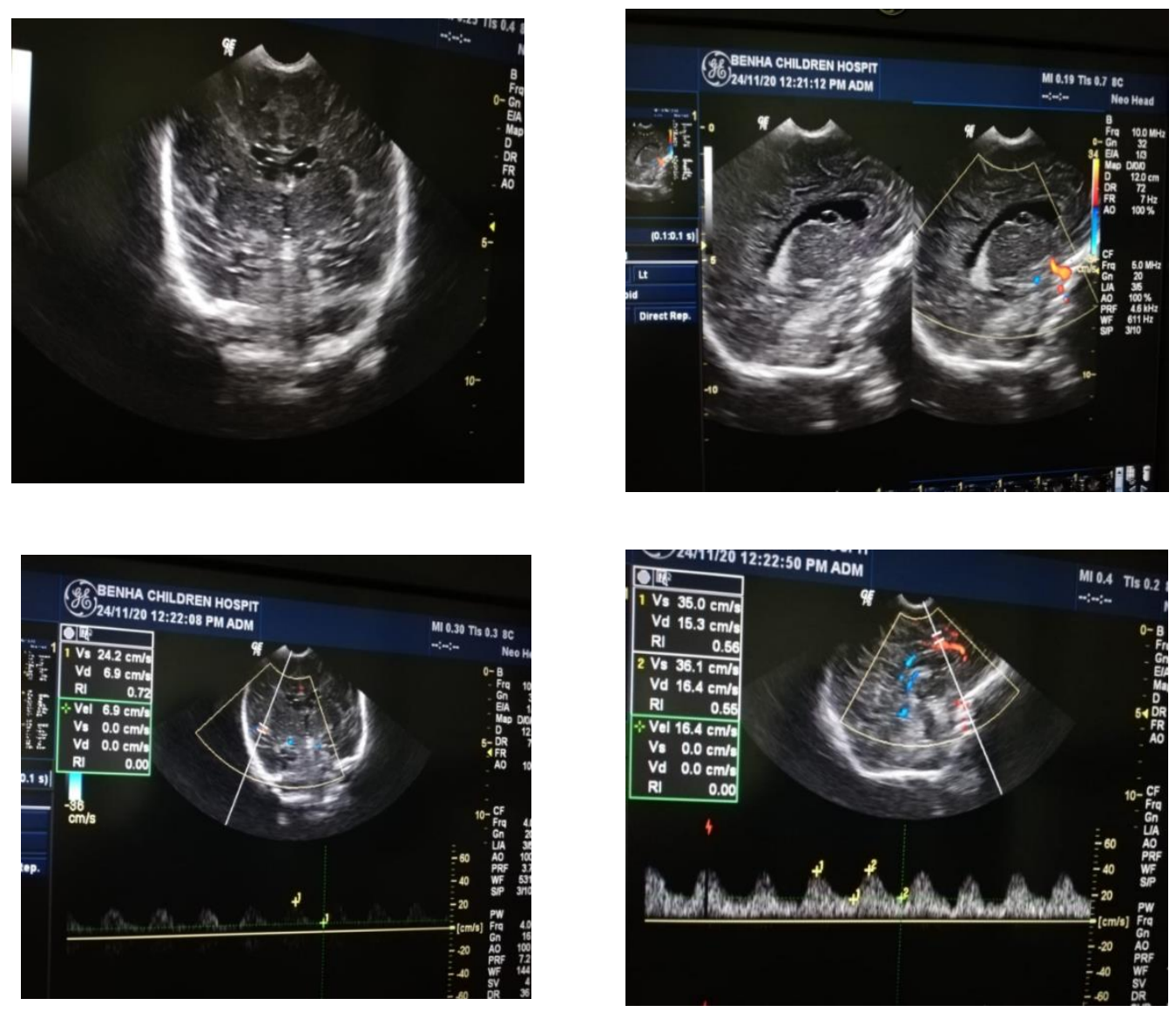

Fig (5) - Case 3: Anterior choroidal simple Cyst 

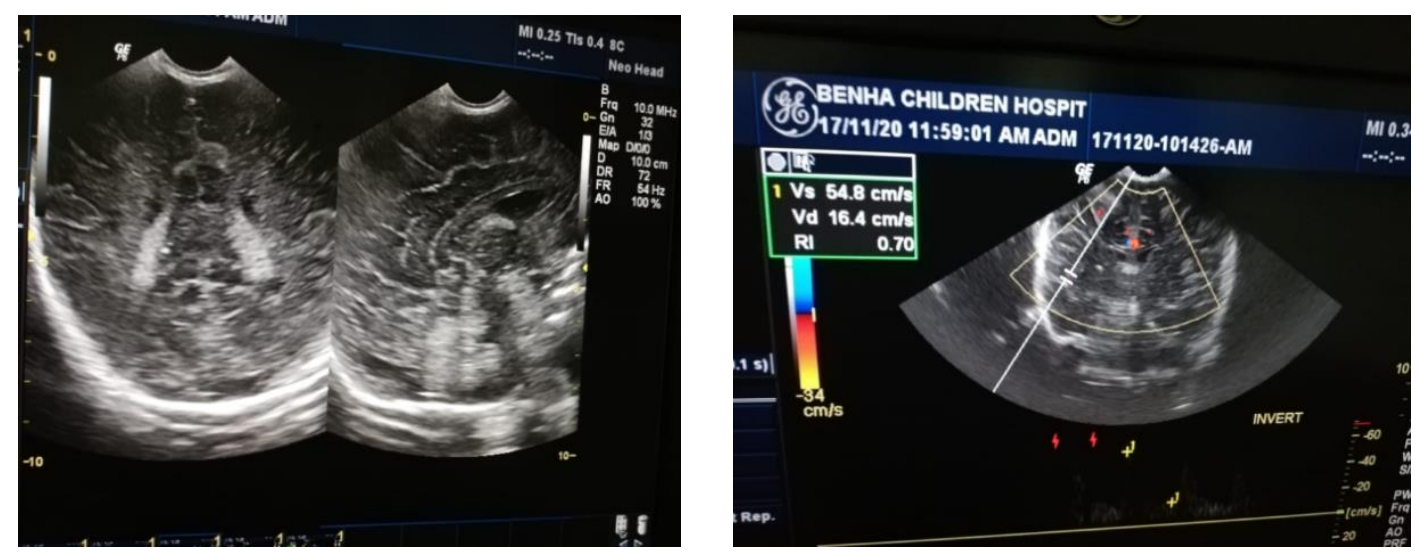

Fig (6) - Case 4: Normal cranial US

\section{Discussion:}

Cranial ultrasound is comparatively inexpensive, does not need sedation or radiation, and provides the significance benefit of being portable. Sequential CUS is the standard imaging modality in the preterm neonates. It can certainly detect germinal matrix, intraventricular hemorrhage, cystic periventricular leukomalacia, ventricular dilatation, and post-hemorrhagic hydrocephalus. It is inexpensive, repeatable, and allows persistent bedside monitoring of $\mathrm{CBFV}$, which is especially beneficial in the intensive care setting. Transcranial Doppler (TCD) ultrasonography is a technique that uses a hand-held Doppler transducer (placed on the surface of the cranial skin) to measure the velocity and pulsatility of blood flow within the intracranial and the extracranial arteries.

In spite of the improvements in perinatal medicine, brain injury is still a major clinical problem and remains an important reason of perinatal morbidity and mortality.

The significance of preterm screening by cranial ultrasound is preserved by the observation that most preterm neonates with abnormal CUS are usually asymptomatic. Only occasionally these patients develop symptoms (seizures or other neurological symptoms).

The preterm neonates. It can reliably detect germinal matrix, intraventricular hemorrhage, cystic periventricular 
leukomalacia, ventricular dilatation, and post-hemorrhagic hydrocephalus. Germinal matrix hemorrhage $(\mathrm{GMH})$ is a recurrent finding in the neonatal period. It occurs primarily, but not exclusively, in $\mathrm{PT}$ neonates of very low birth weight. One of the main problems in preterm neonates is damage to white matter. This damage involves multifocal necrosis resulting in cystic periventricular leukomalacia (PVL) or a diffuse astrogliosis and loss of myelinproducing oligodendrocytes. PVL frequently exists together with intraventricular hemorrhages, this reflecting the vulnerability of the pre myelinating oligodendrocytes. Congenital brain anomalies could be seen during the cranial ultrasound screening, such as Dandy-Walker malformation (DWM), Chiari II malformation, agenesis of corpus callosum.

Preterm neonates less than 37 weeks of gestational age are more susceptible to hemorrhagic and ischemic brain lesions because of the immaturity of capillary bed, cerebral auto-regulation and vascular anastomosis, with consequent adverse outcome, poor cognitive development, and cerebral palsy.

Hemorrhagic brain lesions (GMH and IVH) were the most frequent finding. Ischemic brain insults (PVL) were also detected. Two comparatively common congenital brain anomalies were detected (DWM and complete agenesis of $\mathrm{CC}$ ) in addition to congenital porecephalic cyst. Mild grades of GMH and PVL showed regressive course while the severe grades of GMH deteriorated clinically and died. Linear high frequency probe of ultrasound was of major benefit in better visualization of anatomical details and mild pathological lesions. Small number of the study population, short study time and limited follow up were the main limitations of this study.

TCD examinations have acquired a significant role in the very early phase of critical cerebral pathologies, also during follow-up of patients with chronic CVDs. It is also beneficial on both adults and children to diagnose and monitor vasospasm (VSP) after subarachnoid hemorrhage (SAH) of various etiologies (aneurysm rupture and traumatic brain injury [TBI]), and cerebral hemodynamic changes after stroke including cryptogenic stroke. It allows to investigate cerebral pressure autoregulation and for the clinical evaluation of cerebral auto regulatory reserve. 


\section{Conclusion}

Doppler cranial ultrasound was proven to have a significant role in evaluation neonatal brain problems in preterm patients while most of abnormal brain scans are usually asymptomatic patients, particularly which it is considered a safe procedures that doesn't cause non ionizing radiation exposure. As well as, it helps rapid diagnosis without the necessity for prolonged special preparations.

\section{References}

1. Orman G, Benson JE, Kweldam CF, Bosemani T, Tekes A, De Jong MR, et al., Neonatal Head Ultrasonography Today. J Neuroimaging 2014; 00: $1-25$.

2.Saqqur M, Zygun D, Demchuk A. Role of transcranial Doppler in neurocritical care. Crit Care Med. 2007; 35(5): 216-23.
3. Vijay MD, Georgios MD, Annabell MD, Andrei MD. Role of transcranial Doppler ultrasonography in evaluation of patients with cerebrovascular disease. Current Neurology and Neuroscience Reports 2007; 7: 8-20.

4. Brezan F, Ritivoiu M, Drăgan A, Codreanu I, Răducanu D, Feier D, et al., Preterm screening by transfontanelar ultrasound - results of a 5 years cohort study. Medical Ultrasonography 2012; 14 (3): 204-210.

5. De Vries LS, Gunardi H, Barth PG, Bok LA, Verboon-Maciolek MA, Groenendaal F. The spectrum of cranial ultrasound and magnetic resonance imaging abnormalities in congenital cytomegalovirus infection. Neuropediatrics 2004; 35: 113-119.

6. Christou I, Felberg RA, Demchuk AM, Grotta JC, Burgin WS, Malkoff M, et al. A broad diagnostic battery for bedside transcranial Doppler to detect flow changes with internal carotid artery stenosis or occlusion. J Neuroimaging. 2001;11: 236-42.

7. Iliodromiti Z, Zygouris D, Karagianni P, Belitsos P, Daniilidis A, Vrachnis N: Brain Injury in Preterm Infants. ISBN 978-953-51-0692-0. 2012.

To cite this article: Ahmed F. Yousef, Hamada M. Khater, Shiamaa H. Abdel Bedea. Role of Transcranial Ultrasound Augmented by Color Doppler Study in Evaluation Neonatal Brain Problem in Preterm Patient. BMFJ 2021; 38 (Radiology): 220-231 DOI: 10.21608/ bmfj. 2021. 56774. 1368 